\title{
AN X-RAY INVESTIGATION OF CRAB-LIKE SUPERNOVA REMNANTS
}

\author{
Z. R. WANG \\ Center of Astron. \& Astrophys., CCAST(World Lab.), Nanjing \\ Department of Astronomy, Nanjing University, China \\ and \\ F. D. Seward \\ Harvard-Smithsonian Center for Astrophysics
}

The Crab Nebula is the most noticeable object in our Galaxy, and the remnant of the famous Chinese guest star appeared in 1054AD, the best association between supernova remants(SNR) and ancient guest stars. Before seventies, the Crab Nebula was considered as a special SNR with different morphology and physical features from that of most SNRs, Now more and more Crab-like SNRs have been detected(Weiler 1985). It is necessary to make a systematical investigation for the Crab-like SNRs, especially for those with central pulsars because they offer us more physical messages than others.

The two associations SNR and PSR, the Crab Nebula with PSR 0531+21 and the Vela SNR with PSR 0833-45, were confirmed at the end of sixties and still were the only two associations before eighties. Recent years, PSR 1509-58 in MSH 15-52 and PSR 0540-69 in a SNR of LMC(Seward \& Harnden 1984, Seward etal. 1984) as well as PSR 1951+32 in CTB 80 ( Clifton et al. 1987, Fruchter etal. 1987 ) were discovered. On the other hand, the X-ray emission of PSR 1055-52 and others were detected (Cheng \& Helfand 1983, Helfand 1983). Recently, we searched for the X-ray emission of isolated pulsars over more than thirty fields in Einstein observation. By use of the same instrument calibrstions, the $\mathrm{X}$-ray luminosities of ten isolated pulsars and their surrounding nebulae are obtained. Let $\mathrm{L}_{\mathrm{x}}$ be the total $\mathrm{X}$-ray luminosity of a pulsar and its nebula in the 0.2-4.0 kev band, and $\dot{E}$ be the rotating energy loss rate of the pulsar, the following statistical relation is obtained by linear regression and showr as the line in Fig. 1.

$$
\operatorname{LOG} L_{x}=1.30 \text { LOG } \dot{\mathrm{E}}-13.2
$$

It strongly supports that the energy source of X-rays both from an isolated pulsar and its nebula come from the rotating energy loss of the pulsar, and the energy transformation may be simply and directly. Eq.(1) offers a good empirical relation to further investigate their $\mathrm{X}$-ray radiation mechanism.

Besides the above Crab-like SNRs with PSRs, ther are some Crab-like SNRs with central compact sources but no pulsating radiation being observed, such as 3C 58 ,G 21.5 -0.9 , Kes 73 and Kes 75 et al.. If we assume that such compact sources are also pulsars in nature but only hidden and $\mathrm{Eq}(1)$ is approximately satisfied for them, their periods 


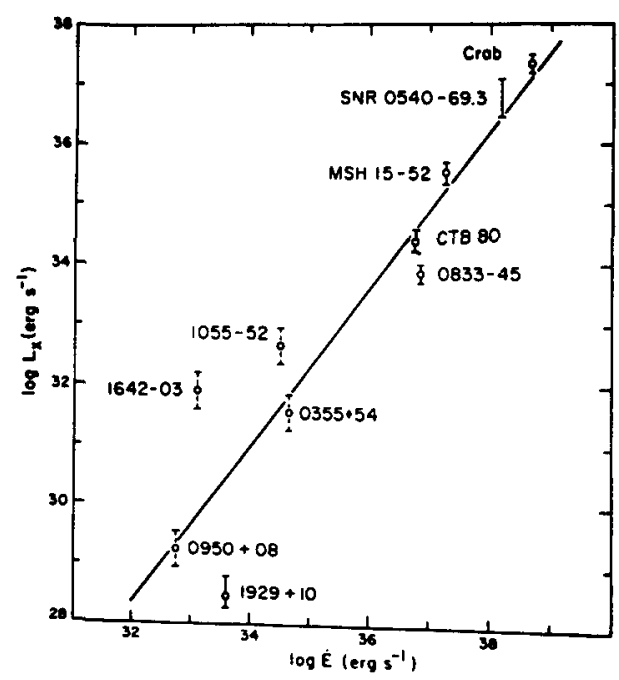

Figure 1.

can be deduced from their X-ray luminosities and their ages which are estimated from their identification with guest stars (Wang et a1.1986, Wang 1987). Some of the pulsed signals will be expected by the future observations with higher resolution and sensitivity.

\section{References}

Cheng, A.F. and Helfand, D.J., 1983, Ap.J., 271, 271

Clifton, T.R. etal., 1987, IAU Circ. No. 4422

Fruchter, A.S. et al., 1987, , IAU Circ. No.4426

Helfand, D.J., 1983, in Supernova Remnants and Their X-Ray Emission, Danziger, J. and Gorenstein, P. Eds. (Dordrecht Reidel) , 471

Seward, F.D. and Harnden, Jr. F.R., 1984, Ap.J., 281, 650

Seward, F.D. Harnden, Jr. F.R. and Helfand, D.J., 1984, Ap.J., 287, L19

Strom, R., 1987, Ap. J. in press

Wang, Z.R., Liu, J.Y., Gorenstein, P. and Zombeck, M.V., 1986, in Highlights of Astronomv, 7, Swings, J.P. Ed., 583

Wang, Z.R., 1987, in The Origin and Evolution of Neutron Stars, Helfand, D.J. and Huang, J. Eds., (Dordrecht Reidel), 305

Weiler, K.W., 1985, in The Crab Nebula and Related Supernova Rermants, Kafatos, M.and Henry, R. Ed. (Cambridge Univ. Press, London) 